\title{
LATTICE VIBRATIONS OF THE MOLECULAR CRYSTAL OF PHOTOREACTIVE SUBSTANCE WITH DEFECTS
}

\author{
Y. Syetov \\ Oles Honchar Dnipro National University, Dnipro, Ukraine \\ e-mail:setov2003@yahoo.com
}

Lattice vibrations are studied theoretically by density-functional based tight-binding methods for the model structure of 2-(2'-hydroxyphenyl)benzoxazole crystal with defects. 2-(2'-hydroxyphenyl)benzoxazole is a photoreactive compound that exhibits excited state intramolecular proton transfer in the structure with an $\mathrm{OH} . . . \mathrm{N}$ hydrogen bond. The unit cell of the model structure consists of two crystal unit cells where the molecules have the structure with intramolecular hydrogen bonds $\mathrm{OH}$...N and one molecule is supposed to have a different orientation of the whole molecule or its fragment. The different orientation of the fragment forms the structure with an intramolecular hydrogen bond OH...O. It is calculated that defect caused by the different orientation of the molecule have a lower energy than the defect caused by the different orientation of the fragment. In the frequency region where the contribution of external vibrations of the molecules is significant, the vibrations mainly involve several molecules in the cell. In the region of internal vibrations there are modes, which are local vibrations of the defects. These local vibrations involve mainly motion of the atoms constituting the defect molecule. The number of local vibrations is larger for the defect that corresponds to the formation of the structure with the $\mathrm{OH} . . . \mathrm{O}$ hydrogen bond than for the defect that corresponds to the different orientation of the whole molecule with the OH...N hydrogen bond. The internal vibrations of the defect molecule formed by the different orientation of phenol fragment in the lattice undergoes frequency shift in relation to the frequency of the modes of isolated molecule.

Keywords: molecular crystal, excited state proton transfer, vibrations, defects, density-functional based tight-binding method.

Received 02.11.2019; Received in revised form 11.12.2019; Accepted 23.12.2019

\section{Introduction}

Photoinduced excited state intramolecular proton transfer (ESIPT) is studied for several decades as an ultrafast photochemical reaction and a process that could be utilized for a number of applications in optoelectronics [1]. 2-(2'-hydroxyphenyl)benzoxazole (HBO) is a compound that undergoes photoinduced excited state intramolecular proton transfer (ESIPT) from oxygen to nitrogen atom. A molecule of $\mathrm{HBO}$ might have several intramolecularly $\mathrm{H}-$ bonded enol structures which differ by mutual orientation of the benzoxazole and phenol moieties (Fig. 1a). Only the form with the OH...N hydrogen bond I demonstrates ESIPT and fluorescence with anomalously large Stokes shift. Conformational disorder (approximately 1:1 ratio of structures I and II) has been determined by the X-ray study of HBO [2], however, only the fluorescence band with a large Stokes shift is observed for crystalline HBO [3]. The IR spectra in the region of intramolecular vibrations of $\mathrm{HBO}$ do not demonstrate bands which can be assigned to the vibrations of structures with $\mathrm{OH}$...O hydrogen bond [4]. In the previous study [5] calculations of the energy of model molecular clusters, which correspond to an arrangement of the molecules in the crystal, show that the defect formed by the molecule rotation has a lower energy than the defect formed by the rotation of molecular fragments. However, in the paper [5] the environment of the defect is assumed to be rigid. In the present paper we describe results of theoretical study of energy and vibrations of a lattice formed by molecules of $\mathrm{HBO}$ with conformational and orientation defects.

\section{Calculations details}

The structure and vibrational modes of model structures of an HBO crystal were calculated by density functional based tight-binding (DFTB) method with the third order correction and van der Waals interaction correction implemented in the DFTB+ program package [6,7] as described in [8]. A $2 \times 2 \times 2$ supercell folding was used for the Brillouin zone sampling. 
The structure and phonon calculations were performed for the Brillouin zone center under the quasi-harmonic approximation that assumes the cell parameters fixed to the values taken from the X-ray data [2]. According to the X-ray data [2], HBO crystal belongs to the orthorhombic Pna2 1 structure class with 4 molecules in a unit cell (Fig. $1 b)$.

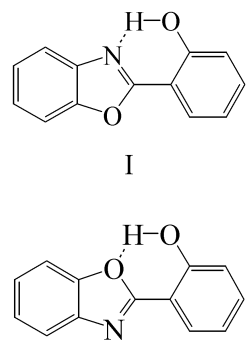

II

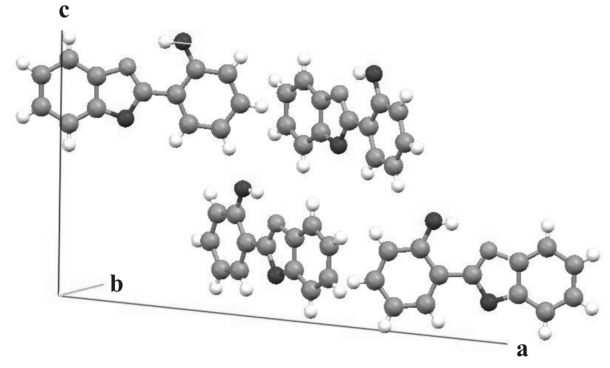

b

Fig. 1. Rotameric structures of molecules (a) and a unit cell of an HBO crystal (b). The unit cell (only rotamers with an $\mathrm{OH}$.... $\mathrm{N}$ hydrogen bond are shown) is plotted according to the $\mathrm{X}$-ray measurements [2].

The model structure of the defect comprises two unit cells of the crystal obtained by translation along the lattice vector $\mathbf{b}$. One molecule in this structure is rotated by approximately $180^{\circ}$ around the long axis or the phenol fragment is rotated around the bond linking the phenol and benzoxazole moieties by $180^{\circ}$ as well (Fig. 2). The structure is assumed to be a unit cell with translation vectors $\mathbf{a}, 2 \mathbf{b}$, and $\mathbf{c}$. The structure is chosen to provide the minimal number of molecules within the first coordination sphere for the defect molecule.

The contribution of the molecule motion to the vibrational modes is estimated as the sum of squared components of the eigenvector of normal vibrations in mass weighted coordinates:

$$
\Delta_{s}=\sum_{i=1}^{N} m_{i}^{s}\left(\xi_{i}^{s}\right)^{2} / \sum_{s=1}^{P} \sum_{i=1}^{N} m_{i}^{s}\left(\xi_{i}^{s}\right)^{2},
$$

where $m_{i}^{s}$ is the mass of the atom $i$ in the molecule $s ; \xi_{i}^{s}$ are components of the normalized eigenvector in Cartesian coordinates; $N$ is the number of atoms in a molecule; $P$ is the number of molecules in a unit cell. For the considered model unit cell $N=25$, $P=8$.

\section{Results and discussion}

The energy of structure II of an isolated molecule of HBO is calculated by DFTB method to be $0.13 \mathrm{eV}$ larger than the energy of structure I. The normal mode calculations for isolated molecules show that there is a difference in frequency for vibrations of structures I and II (Table1). In the case of the model lattice, the lowest energy has the structure with reorientation of the whole molecule (see Fig. 2a). The structure that corresponds to the fully ordered crystal has the energy negligibly (by less than $10^{-3} \mathrm{eV}$ ) larger than the structure with the orientation defect. The energy of the structure with conformational defect (see Fig. 2b) is $0.11 \mathrm{eV}$ larger than for the fully ordered crystal. The energy difference of $0.11 \mathrm{eV}$ implies that the equilibrium concentration of molecules with the different orientation of the phenol fragment according to the Boltzmann distribution is about $10^{-2}$. 


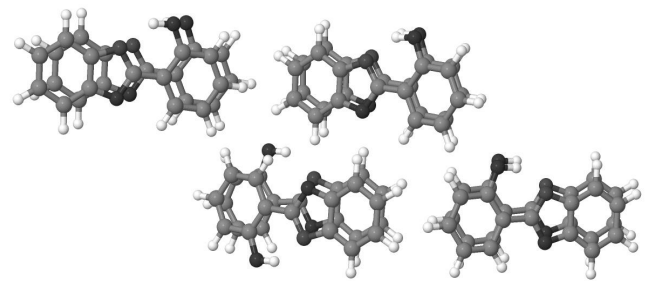

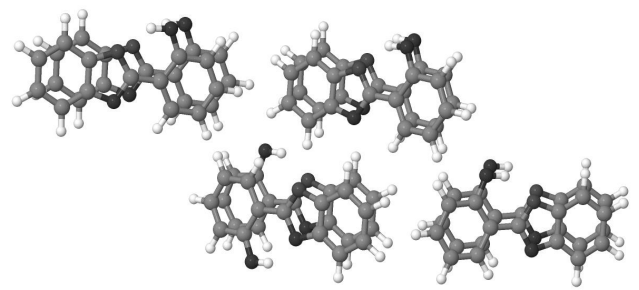

b

Fig. 2. Unit cells of model structures with defects caused by different orientation of the entire molecule (a) and phenol fragment (b) in the crystal lattice of $\mathrm{HBO}$.

Table 1

The calculated frequencies of vibrations of structures I and II of isolated molecules of HBO and local vibrations of structure II in the model lattice

\begin{tabular}{|c|c|c|c|c|c|c|c|c|c|c|c|}
\hline \multicolumn{3}{|c|}{ Frequency, $\mathrm{cm}^{-1}$} & & \multicolumn{3}{|c|}{ Frequency, $\mathrm{cm}^{-1}$} & & \multicolumn{3}{|c|}{ Frequency, $\mathrm{cm}^{-1}$} & \multirow{3}{*}{$\Delta s$} \\
\hline \multicolumn{2}{|c|}{ Structure } & \multirow{2}{*}{$\begin{array}{c}\text { Local } \\
\text { vib. }\end{array}$} & \multirow{2}{*}{$\Delta s$} & \multicolumn{2}{|c|}{ Structure } & \multirow{2}{*}{$\begin{array}{c}\text { Local } \\
\text { vib. }\end{array}$} & \multirow{2}{*}{$\Delta s$} & \multicolumn{2}{|c|}{ Structure } & \multirow{2}{*}{$\begin{array}{c}\text { Local } \\
\text { vib. }\end{array}$} & \\
\hline I & II & & & I & II & & & I & II & & \\
\hline 47 & 35 & & & 743 & 744 & & & 1303 & 1294 & & \\
\hline 60 & 60 & & & 752 & 753 & & & 1340 & 1331 & 1334 & 0.98 \\
\hline 112 & 109 & & & 817 & 817 & & & 1374 & 1359 & 1361 & 0.98 \\
\hline 151 & 147 & 179 & 0.60 & 824 & 827 & 839 & 0.52 & 1423 & 1413 & 1417 & 0.99 \\
\hline 238 & 237 & & & 842 & 839 & 842 & 0.73 & 1451 & 1449 & 1454 & 0.98 \\
\hline 257 & 254 & 267 & 0.77 & 864 & 865 & & & 1510 & 1516 & 1517 & 0.99 \\
\hline 277 & 277 & 286 & 0.51 & 876 & 871 & 871 & 0.66 & 1519 & 1526 & 1527 & 0.99 \\
\hline 311 & 298 & 303 & 0.96 & 885 & 892 & 893 & 0.79 & 1553 & 1546 & 1546 & 0.98 \\
\hline 326 & 325 & 337 & 0.54 & 894 & 895 & & & 1563 & 1562 & 1565 & 0.58 \\
\hline 407 & 407 & & & 900 & 900 & & & 1652 & 1661 & 1662 & 0.92 \\
\hline 458 & 457 & 458 & 0.98 & 922 & 920 & 923 & 0.92 & 1690 & 1692 & & \\
\hline 470 & 460 & 470 & 0.74 & 979 & 949 & 955 & 1.00 & 1723 & 1723 & 1726 & 0.95 \\
\hline 516 & 484 & 513 & 0.92 & 1051 & 1051 & & & 1732 & 1734 & & \\
\hline 537 & 517 & & & 1078 & 1071 & 1077 & 0.92 & 1745 & 1744 & & \\
\hline 562 & 535 & 530 & 0.85 & 1094 & 1082 & 1084 & 0.63 & 3000 & 3000 & 3004 & 0.97 \\
\hline 573 & 561 & 567 & 0.60 & 1131 & 1124 & 1125 & 0.96 & 3001 & 3001 & 3010 & 0.85 \\
\hline 585 & 570 & 573 & 0.75 & 1142 & 1131 & & & 3006 & 3007 & 3017 & 0.73 \\
\hline 588 & 587 & 591 & 0.82 & 1164 & 1163 & 1161 & 0.77 & 3007 & 3009 & 3018 & 0.85 \\
\hline 647 & 645 & 650 & 0.90 & 1169 & 1165 & 1166 & 0.53 & 3016 & 3016 & & \\
\hline 678 & 673 & 676 & 0.84 & 1187 & 1168 & 1172 & 0.82 & 3016 & 3017 & 3025 & 0.93 \\
\hline 691 & 687 & 689 & 0.97 & 1251 & 1241 & 1244 & 0.99 & 3020 & 3020 & 3031 & 0.68 \\
\hline 718 & 712 & 714 & 0.80 & 1281 & 1268 & 1266 & 0.98 & 3023 & 3023 & 3033 & 0.96 \\
\hline 723 & 721 & 726 & 0.82 & 1290 & 1278 & 1281 & 0.85 & 3406 & 3574 & 3568 & 1.00 \\
\hline
\end{tabular}

The number of normal modes of the model lattice with zero wave vectors is 597 . Our analysis of the eigenvectors of the vibrations shows that for frequencies below $155 \mathrm{~cm}^{-1}$ all modes demonstrate contributions $\Delta_{s}<0.5$, so that they involve several molecules. The number of modes in the range below $155 \mathrm{~cm}^{-1}$ is 69 . For the structure that corresponds to the ordered crystal maximum $\Delta_{s}=0.25$ in this frequency region. For the structures with defects the maximum $\Delta_{s}=0.49$ for one vibration. This frequency region comprises lattice vibrations with significant contributions of external motions of the molecules (translations and librations) [8]. In the frequency region of internal vibrations (above $170 \mathrm{~cm}^{-1}$ ) modes with $\Delta_{s}>0.5$ appear. For such modes the vibrations are localized mainly in one molecule. In the case of orientation defect the number of the modes with the motion localized on the defect molecule is 35 , only 9 of which has $\Delta_{s}>0.9$. For the 
structure comprising the molecule with $\mathrm{OH}$... O hydrogen bond the number is 50,23 of which demonstrate $\Delta_{s}>0.9$. Isolated molecule of $\mathrm{HBO}$ is calculated to exhibit 66 normal modes with frequencies above $140 \mathrm{~cm}^{-1}$. The out-of-plane molecular vibration with the calculated frequency of $151 \mathrm{~cm}^{-1}$ (for the structure I with $\mathrm{OH} \ldots \mathrm{N}$ hydrogen bond) undergoes shift in the crystal, giving rise to the lattice vibrations with frequencies about $180 \mathrm{~cm}^{-1}$ [57]. The similar vibration of structure II with the calculated frequency of 147 $\mathrm{cm}^{-1}$ corresponds to a local vibration at $179 \mathrm{~cm}^{-1}\left(\Delta_{s}=0.60\right)$ and noticeably involved into modes $188\left(\Delta_{s}=0.14\right)$ and $189 \mathrm{~cm}^{-1}\left(\Delta_{s}=0.15\right)$ in the model lattice. The other components of the split molecular vibration with the frequencies near $180 \mathrm{~cm}^{-1}$ demonstrate a contribution of the defect molecule less than 0.1. 401 vibrational modes of the model structure are found to have $\Delta_{s}<0.1$ for defect molecule motion. Thus, the internal vibrations of the defect molecule formed by the different orientation of the phenol fragment are mainly localized and undergoes molecule-to-crystal frequency shift. The correspondence of local vibrations of the defect in the model to the vibrations of the isolated molecule with structure II is shown in Table 1.

\section{Conclusions}

Calculations of vibrations of model structures that correspond to an arrangement of the molecules in crystal lattice of 2-(2'-hydroxyphenyl)benzoxazole show that defects caused by a different orientation of a molecule or its fragment lead to the appearance of local vibrations of the defect molecules in the region of internal modes. In the frequency region where contribution of the external vibrations is significant the lattice vibrations are mostly delocalized involving several molecules. The calculations show that due to the shape and crystal package of HBO molecules the energy of the defect cased by reorientation of the molecule about its long axis is lower than the energy of the defect formed by the phenol fragment reorientation.

\section{References}

1. Hsieh, C.-C. Organic Dyes with Excited-State Transformations (Electron, Charge, and Proton Transfers) / C.-C. Hsieh, M.-L. Ho, P.-T. Chou // Advanced Fluorescence Reporters in Chemistry and Biology I: Fundamentals and Molecular Design / Ed. by A.P. Demchenko. - Springer Ser. Fluoresc. - 2010. - Vol. 8. - P. 225 - 266.

2. Tong, Y.-P. 2-(2-Hydroxyphenyl)-1,3-benzoxazole / Y.-P. Tong // Acta Cryst. E. - 2005. - Vol. 61. - P. o3076 - o3078.

3. Syetov, Y. Luminescence spectrum of 2-(2'-hydroxyphenyl)benzoxazole in the solid sate / Y. Syetov // Ukr. J. Phys. Opt. - 2013. - Vol. 14, No. 1. - P. 1 - 5.

4. Syetov, Y. Infrared spectra of benzoxazoles exhibiting excited state proton transfer / Y. Syetov, A. Vdovin // Vib. Spectrosc. - 2010. - Vol. 53. - P. 146 - 150.

5. Syetov, Y. Lattice vibrations and disorder in crystalline benzoxazoles undergiong exited state intramolecular proton transfer: DFTB modeling / Y. Syetov // Journal of Physics and Electronics. - 2018. - Vol. 26(1). - P. 57 - 62.

6. Aradi, B. DFTB+, a Sparse Matrix-Based Implementation of the DFTB Method / B. Aradi, B. Hourahine, Th. Frauenheim // J. Phys. Chem. A. - Vol. 111. - 2007. - P. 5678 - 5684.

7. Gaus, M. DFTB3: Extension of the Self-Consistent-Charge Density-Functional Tight-Binding Method (SCC-DFTB) / M. Gaus, Q. Cui, M. Elstner // J. of Chem. Theory Comput. - 2011. - Vol. 7. - P. 931 - 948.

8. Syetov, Y. Low-frequency Raman spectrum of crystalline 2-(2'hydroxyphenyl)benzoxazole and density-functional based tight-binding phonon calculations / Y. Syetov // Ukr. J. Phys. Opt. - 2017. - Vol. 18, No. 2. - P. 67 - 76. 